\title{
A phase I study of tasisulam sodium (LY573636 sodium), a novel anticancer compound in patients with refractory solid tumors
}

\author{
George R. Simon • Robert L. Ilaria Jr. • Mika A. Sovak • Charles C. Williams • Eric B. Haura • \\ Ann L. Cleverly • Amanda K. Sykes • Margaret M. Wagner · Dinesh P. de Alwis • \\ Christopher A. Slapak • Mary A. Miller · David R. Spriggs
}

Received: 15 November 2010 / Accepted: 17 February 2011 / Published online: 23 March 2011

(C) The Author(s) 2011. This article is published with open access at Springerlink.com

\begin{abstract}
Purpose This phase I study was carried out to determine the phase II recommended dose of tasisulam sodium (hereafter, tasisulam), a novel anticancer agent with a unique mechanism of action.

Methods Tasisulam was administered intravenously, every 21 days, in patients with refractory solid tumors using a three-plus-three dose-escalation schema.

Results Fifty-three patients were enrolled; the first 34 were treated with a flat dose of tasisulam of up to $2,400 \mathrm{mg}$, the dose level at which all three patients had dose-limiting toxicity (DLT). Controlling for $C_{\max }$ proved important to reduce the risk of toxicity; therefore, we initially focused on identifying which parameters explained $C_{\max }$
\end{abstract}

This study was presented in part at the 43rd American Society of Clinical Oncology Annual Meeting, June 1-5, 2007, Chicago, IL; the 16th European Organisation for Research and Treatment of Cancer-National Cancer Institute-American Association for Cancer Research Annual Symposium on Molecular Targets and Cancer Therapeutics, Sept. 28-Oct. 1, 2004, Geneva, Switzerland; and the 22nd European Organisation for Research and Treatment of Cancer-National Cancer Institute-American Association for Cancer Research Annual Symposium on Molecular Targets and Cancer Therapeutics, Nov. 16-19, 2010, Berlin, Germany.

G. R. Simon · C. C. Williams · E. B. Haura

H. Lee Moffitt Cancer Center, Tampa, FL, USA

R. L. Ilaria Jr. · M. M. Wagner · C. A. Slapak · M. A. Miller

Eli Lilly and Company, Indianapolis, IN, USA

A. L. Cleverly · A. K. Sykes · D. P. de Alwis

Eli Lilly and Company, Windlesham, Surrey, UK

M. A. Sovak · D. R. Spriggs

Memorial Sloan Kettering Cancer Center,

New York, NY, USA (end-of-infusion concentration) variability. Pharmacokinetic analysis indicated that $C_{\max }$ negatively correlates with lean body weight (LBW). Thus, the dosing regimen was revised using a LBW-based algorithm targeting a specific $C_{\max }$. A loading/chronic dose paradigm was then implemented as pharmacokinetic results revealed a long terminal half-life of tasisulam, likely because of its high-affinity albumin binding. $C_{\max }$-based dose escalation was stopped at the $420-\mu \mathrm{g} / \mathrm{mL}$ cohort, in which one of the 16 patients had DLT (transient hepatic transaminase elevation); grade $3 / 4$ hematologic toxicity was noted in later cycles in three patients. Although response was not a primary objective, $33 \%$ of heavily pretreated patients with post-dose radiological assessments had stable disease.

Conclusion Implementation of a novel targeted $C_{\max }$ based dosing regimen allowed for the recommendation of a phase II tasisulam dose (loading dose of $420 \mu \mathrm{g} / \mathrm{mL}$ targeted $C_{\max }$ with all subsequent doses administered at $65 \%$ of chronic dose given every 21 days) despite pharmacological challenges posed by high albumin binding.

Keywords Tasisulam $\cdot$ Solid tumors $\cdot$ Phase I · Chemotherapy $\cdot$ LY573636

Present Address:

G. R. Simon $(\square)$

The Burtschy Family Endowed Chair in Cancer Research, Division of Hematology/Oncology, Department of Medicine, Medical University of South Carolina, 96 Jonathan Lucas Street, Suite 903, MSC 635, Charleston, SC 29425-6350, USA e-mail: simong@musc.edu

Present Address:

M. A. Sovak

Genentech, South San Francisco, CA, USA 


\section{Introduction}

Tasisulam sodium (benzamide, $N$-[(5-bromo-2-thienyl)sulfonyl]-2,4-dichloro-, sodium salt; hereafter referred to as tasisulam), an acylsulfonamide, is a member of a new class of antineoplastic agents [1]. An in vitro, antitumor-screening analysis conducted by the National Cancer Institute (NCI COMPARE analysis) [2] demonstrated a broad range of activity for tasisulam in 60 tumor cell lines including leukemia, melanoma, non-small cell lung (NSCLC), colon, ovarian, renal, and breast cancers. In addition, the activity profile of tasisulam in the COMPARE analysis did not match that of any other known anticancer compound [2], which suggests a mechanism of action for tasisulam that is distinct from currently available agents.

Preclinical in vitro studies indicate that tasisulam induces apoptosis in cancer cells by the mitochondrial (intrinsic) cell death pathway [3], and also induces a block at the G2/M phase of the cell cycle. However, the precise cellular or mitochondrial target that leads to the induction of apoptosis remains unknown. Tasisulam has also demonstrated anti-angiogenic activity in vitro (Lilly data on file).

In vivo pharmacokinetic studies in rats and dogs indicate that tasisulam is metabolized primarily by the liver, and has low total plasma clearance with a relatively long half-life (approximately $10 \mathrm{~h}$ in rats and $20 \mathrm{~h}$ in dogs). In vitro measurements also showed that tasisulam is highly protein bound ( $\sim 99 \%)$ in humans, mice, rats, and dogs (Lilly data on file). In addition, there was preclinical evidence of a correlation between the maximum plasma concentration $\left(C_{\max }\right)$ of tasisulam and toxicity (Lilly data on file).

The broad spectrum of preclinical antitumor activity of tasisulam and its potentially unique mechanism of action prompted this phase I dose-escalation study in patients with advanced or refractory solid tumors. The primary objective of the study was to determine the recommended phase II dose of tasisulam; secondary objectives were to characterize the toxicity and pharmacokinetic profiles of tasisulam, and to discern whether there was any antitumor activity.

\section{Patients and methods}

\section{Eligibility criteria}

Patients $\geq 18$ years of age with histologically or cytologically documented advanced and/or metastatic malignancy for which no proven effective therapy exists were eligible to participate in this study. Patients were required to have a performance status of 0 or 1 on the Eastern Cooperative Oncology Group (ECOG) scale, adequate bone marrow reserve, kidney and liver function. Discontinuation of all the previous therapies (including chemotherapy, radiotherapy, or other investigational therapy) at least 4 weeks before study enrollment and recovery from all toxicities were also required. Patients who had hematologic malignancies or a history of central nervous system neoplasm or who required palliative radiotherapy at study entry were excluded from study participation.

Written informed consent was obtained from each patient before study participation. The study was approved by the institutional review board(s) of the participating institutions, and was conducted according to applicable laws and regulations, good clinical practices, and the ethical principles of the Declaration of Helsinki.

\section{Treatment plan and dose-escalation schema}

For the purpose of this open-label, single-arm, dose-escalating, phase I study, tasisulam was supplied by Eli Lilly and Company (Indianapolis, IN) in single-use, 20-mL glass vials containing tasisulam sodium, sodium chloride, and water or as a freeze-dried formulation in a 50-mL-vial equivalent to $500 \mathrm{mg}$ of base compound. Tasisulam sodium was diluted in $500 \mathrm{~mL}$ of $0.9 \%$ sodium chloride, and was stored at room temperature for up to $8 \mathrm{~h}$. This compound was administered as an intravenous infusion over $2 \mathrm{~h}$ ( $\pm 10 \mathrm{~min})$ on day 1 of a 21 -day cycle.

Dose escalation and the maximum tolerated dose (MTD) were based on toxicity and pharmacokinetic results observed in cycle 1. Dose-limiting toxicity (DLT) was defined as possibly drug-related grade $\geq 3$ non-hematologic toxicity (except for nausea/vomiting without maximal symptomatic/prophylactic treatment and alopecia) according to the NCI Common Toxicity Criteria (CTC) version 2.0 or grade 4 hematologic toxicity of $>5$ days duration.

Initially, dose escalation was based on a flat-dose escalation schema ranging from 100 to $2,400 \mathrm{mg}$ (100, 200, 400, 660, 1,000, 1,400, 1,800, and 2,400 mg). Early in the study, intrapatient dose escalation to the next higher cohort was permitted if a patient completed two cycles of treatment without a DLT; however, the toxicities of these patients were not included in the DLT evaluation. The flat-dose escalation schema was later revised to one based on lean body weight (LBW; discussed further in "Results").

The dose-escalation schema followed a three-plus-three design [4]. Per the protocol, if $\geq 2$ patients in a cohort had DLT, then the MTD was surpassed and dose escalation was stopped. The MTD was then identified as the next lower cohort in which less than two of six patients had DLT, but if the dose exposure was nonlinear, patient recruitment was allowed into additional cohorts between the highest cohort reached and the previous cohort evaluated to identify the recommended phase II dose. 
Baseline and treatment assessments

Medical history, physical examination, performance status assessment, and tumor measurement of palpable or visual lesions were all carried out at baseline and at every cycle. Complete blood counts, serum chemistry, and urinalysis were performed at baseline, before each cycle, and posttherapy. Twelve-lead electrocardiograms were conducted at baseline, during cycle 1, and post-therapy. Clinically indicated radiologic imaging (computerized tomography scan, magnetic resonance imaging, or chest radiograph) was performed at baseline and at every other cycle to assess response to treatment. Tumor responses were followed and recorded using Response Evaluation Criteria in Solid Tumors (RECIST) [5]; however, the study was not designed to assess treatment efficacy.

\section{Bioanalytical methods}

Plasma samples containing ethylenediaminetetraacetic acid (EDTA) were analyzed for total tasisulam concentrations using two validated liquid chromatography-tandem mass spectrometry (LC-MS/MS) methods. A dual-range method (that is, high-range and low-range) was needed due to the large peak to trough plasma concentrations observed. The calibration range for the high-range method was 2.50$500.0 \mu \mathrm{g} / \mathrm{mL}$, and for the low-range method, it was $0.025-$ $5.00 \mu \mathrm{g} / \mathrm{mL}$. Samples above the upper limit of quantification were diluted and reanalyzed to yield results within the calibrated range. Samples below the lower limit of quantification using the high-range method were reanalyzed using the low-range method. Tasisulam and internal standard were extracted from EDTA plasma by protein precipitation using a 96-well plate format.

For the high-range method, accuracy (\% relative error) for the original validation ranged from -3.73 to $12.52 \%$ for the intra-assay accuracy and between -1.93 and $8.36 \%$ for the inter-assay accuracy. Precision (\% relative standard deviation) ranged from 1.18 to $6.72 \%$ for intra-assay precision and between 2.095 and $7.27 \%$ for inter-assay precision.

For the low-range method, accuracy (\% relative error) for the original validation ranged from -2.88 to $13.84 \%$ for the intra-assay accuracy and between 5.77 and $7.69 \%$ for the inter-assay accuracy. Precision (\% relative standard deviation) was between 2.38 and $11.54 \%$ for the intra-assay precision and between 0.760 and $8.080 \%$ for the inter-assay precision.

\section{Pharmacokinetic evaluations}

Blood samples were collected predose, 0.5, 1, 2 (end of infusion), 2.25, 2.5, 3, 4, 5, 6, 8, 12, 24, 48, 72, and $168 \mathrm{~h}$
(7 days) after the start of the infusion to evaluate the pharmacokinetic parameters of tasisulam. In addition, EDTA plasma samples (approximately $7 \mathrm{~mL}$ ) were obtained predose from the first 12 patients in the study to assess protein binding. Pharmacokinetic parameters were computed by standard noncompartmental methods using WinNonlin Enterprise 5.0.1.

\section{Statistical methods}

Dose proportionality was assessed by fitting the power model to pharmacokinetic parameters against dose and summarized using the methods described by Smith et al. [6]. Body-size parameters were added to the power model to determine which of these best explained the variability in pharmacokinetic parameters. The choice was based on selecting the model with the smallest Akaike Information Criterion (AIC) [7] for those covariates that significantly reduced the underlying interpatient variability.

\section{Results}

Patient characteristics

This study was conducted at the H. Lee Moffitt Cancer Center, Tampa, FL and the Memorial Sloan-Kettering Cancer Center, New York, NY, in collaboration with Eli Lilly and Company. From August 2003 to February 2007, 62 patients were entered into the study and 53 received at least one dose of tasisulam (seven patients did not meet enrollment criteria and two withdrew consent). Patient demographics are summarized in Table 1.

\section{Dose-escalation}

On the basis of preclinical toxicity and efficacy testing (Lilly data on file), tasisulam was administered as a flat dose, ranging from 100 to $2,400 \mathrm{mg}$, to 34 patients enrolled in cohorts 1 through 9 (Table 2). DLTs were not observed in patients enrolled in cohorts 1 through $6(100-1,400 \mathrm{mg})$. One of the three patients treated in cohort $7(1,800 \mathrm{mg})$ was discontinued from the study in cycle 1 because of tumorrelated spinal cord compression and was replaced; this patient had a grade 3 DLT (diarrhea). Three additional patients were then enrolled in this cohort (for a total of seven patients), and no additional DLTs were observed. In cohort $8(2,400 \mathrm{mg})$, all three enrolled patients experienced DLT. One patient with metastatic NSCLC and a history of inflammatory colitis developed grade 3 stomatitis, grade 3 thrombocytopenia, and gastrointestinal bleeding, and later developed grade 4 febrile neutropenia and fatal acute respiratory distress syndrome (ARDS) in the setting of bilateral 
Table 1 Baseline patient demographics and disease characteristics $(N=53)$

\begin{tabular}{|c|c|}
\hline \multicolumn{2}{|l|}{ Sex, $n(\%)$} \\
\hline Female & $37(69.8)$ \\
\hline Male & $16(30.2)$ \\
\hline \multicolumn{2}{|l|}{ Ethnicity, $n(\%)$} \\
\hline Caucasian & $47(88.7)$ \\
\hline Hispanic & $3(5.7)$ \\
\hline African descent & $2(3.8)$ \\
\hline East/Southeast Asian & $1(1.9)$ \\
\hline \multicolumn{2}{|l|}{ Age, years } \\
\hline Median & 57 \\
\hline Range & $18-83$ \\
\hline \multicolumn{2}{|l|}{ ECOG performance status, $n(\%)$} \\
\hline 0 & $28(52.8)$ \\
\hline 1 & $22(41.5)$ \\
\hline 2 & $2(3.8)$ \\
\hline Unknown $^{\mathrm{a}}$ & $1(1.9)$ \\
\hline \multicolumn{2}{|l|}{ Tumor sites/histology, $n(\%)$} \\
\hline Lung $^{\mathrm{b}}$ & $14(26.4)$ \\
\hline $\operatorname{Sarcoma}^{\mathrm{c}}$ & $10(18.9)$ \\
\hline Carcinoma $^{\mathrm{d}}$ & $8(15.1)$ \\
\hline Ovarian & $6(11.3)$ \\
\hline Melanoma & $3(5.7)$ \\
\hline Colon & $2(3.8)$ \\
\hline Breast & $2(3.8)$ \\
\hline Other $^{\mathrm{e}}$ & $8(15.1)$ \\
\hline Median no. prior medications (range) ${ }^{\mathrm{f}}$ & $6(1-16)$ \\
\hline
\end{tabular}

ECOG Eastern Cooperative Oncology Group

${ }^{\text {a }}$ Patient was subsequently identified as having a performance status of 0 at baseline

b Non-small cell lung cancer (4 patients), adenocarcinoma (4 patients), mesothelioma (4 patients), bronchioloalveolar carcinoma (1 patient), and squamous (1 patient)

${ }^{c}$ Sarcoma (4 patients), uterine leiomyosarcoma (3 patients), osteogenic sarcoma ( 2 patients), and angiosarcoma (1 patient)

${ }^{\mathrm{d}}$ Carcinoma (2 patients), thyroid carcinoma medullary (2 patients), adenocarcinoma unknown primary (1 patient), adenoid cyst carcinoma (1 patient), adrenal gland carcinoma (1 patient), and cholangiocarcinoma (1 patient)

e One patient each: endometrial, endocervical, bladder, pancreas, papillary adenocarcinoma, parotid gland, thymoma, and urothelium

${ }^{\mathrm{f}} N=52$; prior medication(s) in one patient was not available

pulmonary nodules and pleural effusions. Another patient developed a prolonged prothrombin time (16-day duration without significant bleeding) that was later attributed to an interaction between tasisulam and the concomitantly administered medication, warfarin. The third patient developed hemoptysis from a tracheostomy site and grade 3 bilateral pneumonia 3 days after administration of tasisulam; the patient's white blood cell count was $12.4 \mathrm{k} / \mu \mathrm{L}$ and platelet count was $159 \mathrm{k} / \mu \mathrm{L}$. Bronchoscopy was negative for active bleeding, and no further hemoptysis was noted, but secretions were considered consistent with alveolar hemorrhage (grade 3 pulmonary hemorrhage). Because the MTD had been surpassed at the 2,400-mg flat-dose cohort, three patients were enrolled at an intermediate flat dose of 2,000 mg (cohort 9), and three additional patients were enrolled at the 1,800-mg flat dose (cohort 7). No additional DLTs were observed.

One patient each in cohorts 7 and 9 had grade 4 hematologic toxicity (thrombocytopenia/pancytopenia, respectively) in cycle 2 that lasted at least 8 days in one patient, suggesting the possibility of cumulative toxicity from the accumulation of bound drug with repeated flat dosing.

\section{Pharmacokinetics}

Of the 34 patients administered flat doses of $100-2,400 \mathrm{mg}$ (cohorts 1-9), 33 had full pharmacokinetic profiles from cycle 1 . The analysis of pharmacokinetic data showed high interpatient variability (Table 3), a low total plasma clearance (approximately $0.03 \mathrm{~L} / \mathrm{h}$ ), a volume of distribution that was likely limited to the distribution volume of albumin (approximately 9 L) [8], and a terminal elimination half-life that corresponded to the turnover rate of albumin (approximately 11 days) [9]. Values of $C_{\max }$ increased less than proportionately with dose, with coefficients of variation of $22 \%$ after adjusting for dose (Fig. 1a). On the basis of preclinical animal studies, in which the degree of $C_{\max }$ variability appeared to be associated with the risk of toxicity (Lilly data on file), it was thought that controlling $C_{\max }$ variability in patients should reduce their risk of toxicity. Therefore, the relationship between body size-related parameters and $C_{\max }$ was explored. LBW, which was calculated using an algorithm incorporating the patient's height, weight, and gender [10,11], was identified as an important cofactor that demonstrated a clear inverse relationship between LBW and $C_{\max }$ (Fig. 1b) and reduced the interpatient variability of $C_{\max }$ (from 22 to $13 \%$ ) (Fig. 1c). Thus, pharmacokinetic analysis revealed that the $C_{\max }$ for tasisulam (defined as its concentration at the end of the 2-h infusion) negatively correlated with the LBW of the patient. This led to a change from the flat-dose paradigm to a LBW-based dosing paradigm targeting an individualized $C_{\max }$.

\section{Phase II dose determination}

Although the dose-escalation findings suggested an MTD for tasisulam of approximately $2,000 \mathrm{mg}$, the significant interpatient and intrapatient variability of $C_{\max }$ in the flatdose cohorts and the high degree of albumin binding of tasisulam (99.7-99.9\%) suggested that the flat-dosing schema was unlikely to provide consistent drug exposure. 
Table 2 Dose-limiting toxicity (DLT) and treatment cycles administered by cohort

\begin{tabular}{|c|c|c|c|c|}
\hline \multirow[t]{2}{*}{ Cohort } & \multirow[t]{2}{*}{ Dose } & \multirow[t]{2}{*}{ No. DLTs/No. pts in cycle 1} & \multicolumn{2}{|c|}{ Number cycles administered } \\
\hline & & & Median (range) & Total \\
\hline 1 & $100 \mathrm{mg}$ & $0 / 3$ & $2(2-4)$ & 8 \\
\hline 2 & $200 \mathrm{mg}$ & $0 / 3$ & $2(2-4)$ & 8 \\
\hline 3 & $400 \mathrm{mg}$ & $0 / 3$ & $3(2-4)$ & 9 \\
\hline 4 & $660 \mathrm{mg}$ & $0 / 3$ & $2(1-2)$ & 5 \\
\hline 5 & $1,000 \mathrm{mg}$ & $0 / 3$ & $3(2-4)$ & 9 \\
\hline 6 & $1,400 \mathrm{mg}$ & $0 / 3$ & $2(1-10)$ & 13 \\
\hline 7 & $1,800 \mathrm{mg}$ & 1/10 (G3 diarrhea) & $2(1-8)$ & 28 \\
\hline 8 & $2,400 \mathrm{mg}$ & $\begin{array}{l}\text { 3/3 (G3 stomatitis + gastrointestinal bleeding } \\
\text { + G4 febrile neutropenia; increased PT/INR } \\
\text { for } 16 \text { days }{ }^{\text {a }} \text {; G3 pneumonia } \\
\text { + G3 pulmonary hemorrhage) }\end{array}$ & $1(1-2)$ & 4 \\
\hline 9 & $2,000 \mathrm{mg}^{\mathrm{b}}$ & $0 / 3$ & $2(2-4)$ & 8 \\
\hline 10 & $400 \mu \mathrm{g} / \mathrm{mL}$ & $0 / 3$ & $2(1-6)$ & 9 \\
\hline 11 & $420 \mu \mathrm{g} / \mathrm{mL}$ & $\begin{array}{l}\text { 1/16 (transient grade } 3 \text { hepatic } \\
\text { transaminase elevation) }^{\mathrm{c}}\end{array}$ & $3(1-14)$ & 72 \\
\hline
\end{tabular}

DLT was defined as any drug-related grade $\geq 3$ non-hematologic toxicity according to the NCI Common Toxicity Criteria (CTC) Version 2.0, 1998 (except for nausea/vomiting without maximal symptomatic/prophylactic treatment and alopecia) or grade 4 hematologic toxicity of $>5$ days duration in cycle 1

$G$ grade, $P T / I N R$ prothrombin time/international normalized ratio

a The elevated PT/INR was later attributed to the interaction of tasisulam and the concomitant medication, warfarin; the protocol was subsequently amended to exclude patients who were actively receiving warfarin therapy as tasisulam and warfarin compete for the same albumin binding site

b An intermediate cohort of 2,000 mg (cohort 9) was explored. This cohort represented a dose between 1,800 mg (cohort 7) and 2,400 mg (cohort 8)

c Three additional patients developed hematologic toxicity (grade $3 / 4$ thrombocytopenia/neutropenia) in later cycles $(\geq$ cycle 2 )

These findings led to a change from flat-dosing to a LBWbased dosing paradigm targeting specific $C_{\max }$ values in cohorts 10 and 11 .

Because of the hematologic toxicity observed in later cycles in cohorts 7 and 9, a loading dose was implemented in cycle 1 followed by a lower chronic dose in subsequent cycles in cohorts 10 and 11 to decrease the risk of drug accumulation due to the relatively long terminal half-life of tasisulam. The loading dose in cycle 1 for the LBW-based cohorts targeted a $C_{\max }$ of $400 \mu \mathrm{g} / \mathrm{mL}$ (cohort 10) or $420 \mu \mathrm{g} / \mathrm{mL}$ (cohort 11). The chronic doses were 70 and $65 \%$ of the loading doses for cohorts 10 and 11, respectively. The initial LBW-based $C_{\max }$ dose of $400 \mu \mathrm{g} / \mathrm{mL}$ was considered equivalent to the flat doses that were considered tolerable and showed antitumor activity (disease stabilization).

None of the three patients enrolled in cohort 10 had DLT (Table 2). In cohort 11, one of the six enrolled patients had DLT (transient grade 3 hepatic transaminase elevation); however, two of the six patients developed grade 3 or 4 hematologic toxicity in cycle 2 , suggesting that escalation to a higher targeted $C_{\max }$ was unlikely to identify a dose suitable for administration in phase II. Therefore, cohort 11 was expanded for further study. Of the 16 patients treated in cohort 11, one patient had DLT, and three patients developed grade 3/4 thrombocytopenia/neutropenia in later cycles (cycle 2 or more); thus, dose escalation was stopped.

The median number of treatment cycles in cohort 11 was 3 (range 1-14 cycles) (Table 2). Of the 16 patients in cohort 11,14 completed at least two cycles, and eight completed at least four cycles. Therefore, considering the heavily pretreated patient population and the tolerability of the $420 \mu \mathrm{g} /$ $\mathrm{mL} C_{\max }$ targeted dose, this dose was recommended for phase II study.

All of the 19 patients enrolled in cohorts 10 and 11 had full pharmacokinetic profiles in cycle 1. The pharmacokinetic parameters ( $\mathrm{CL}$ and $\mathrm{V}_{\mathrm{ss}}$ ) were similar in these cohorts compared with those of the flat-dose cohorts 1-9; however, comparison of the 1,800-mg flat-dose group and the 420$\mu \mathrm{g} / \mathrm{mL}$ dose group revealed that the LBW-dosing schema decreased the variability of $\mathrm{AUC}_{(0-\infty)}$ and CL by approximately $50 \%$, and the variability of $C_{\max }$ from 28 to $19 \%$ (Table 3). Comparisons of concentration versus time profiles for six pairs of patients, who were similar in their LBW difference, indicated smaller differences between $C_{\max }$ values in patients dosed to a targeted $C_{\max }$ of $420 \mu \mathrm{g} /$ $\mathrm{mL}$ (Fig. 2a) compared with those given a flat dose (Fig. 2b). 
Table 3 Summary of noncompartmental pharmacokinetic parameters following a 2-h intravenous infusion of tasisulam

\begin{tabular}{|c|c|c|c|c|}
\hline \multirow[t]{2}{*}{ PK parameter } & \multicolumn{4}{|c|}{ Geometric mean $(\mathrm{CV} \%)^{\mathrm{a}}$} \\
\hline & \multicolumn{2}{|c|}{$1,800 \mathrm{mg}(n=8)$} & \multicolumn{2}{|c|}{$\begin{array}{l}420 \mu \mathrm{g} / \mathrm{mL} \\
\text { targeted } C_{\max }(n=13)\end{array}$} \\
\hline$t_{1 / 2}(\mathrm{~h})^{\mathrm{a}}$ & \multicolumn{2}{|c|}{$213(74-942)$} & \multicolumn{2}{|c|}{$269(154-617)$} \\
\hline$C_{\max }(\mu \mathrm{g} / \mathrm{mL})^{\mathrm{b}, \mathrm{c}}$ & \multicolumn{2}{|c|}{$322(28)$} & \multicolumn{2}{|c|}{$359.83(19)$} \\
\hline $\mathrm{AUC}_{(0-\infty)}(\mathrm{h} \mu \mathrm{g} / \mathrm{mL})$ & \multicolumn{2}{|c|}{$70,900(106)$} & \multicolumn{2}{|c|}{$98,100(51)$} \\
\hline $\mathrm{CL}(\mathrm{L} / \mathrm{h})$ & \multicolumn{2}{|c|}{$0.026(107)$} & \multicolumn{2}{|c|}{$0.024(51)$} \\
\hline \multirow[t]{2}{*}{$V_{\mathrm{ss}}(\mathrm{L})$} & & & \multicolumn{2}{|c|}{$9.28(24)$} \\
\hline & & \multicolumn{3}{|c|}{$\begin{array}{l}\text { Arithmetic mean }(\mathrm{CV} \%) \\
\text { (Range) }\end{array}$} \\
\hline LBW (kg) & & $\begin{array}{l}53.8(3 \\
(29.2-8\end{array}$ & & $\begin{array}{l}48.6(22) \\
(35.9-71.7)\end{array}$ \\
\hline \multicolumn{2}{|c|}{ Dose per kg LBW (mg/kg) } & $\begin{array}{l}38.4(3 \\
(20.6-6\end{array}$ & & $\begin{array}{l}49.7(8)^{\mathrm{d}} \\
(42.0-55.6)\end{array}$ \\
\hline
\end{tabular}

$P K$ pharmacokinetic, $C V$ coefficient of variation, $t_{1 / 2}$ terminal elimination half-life, $C_{\max }$ maximum concentration (end-of-infusion concentration), $A U C_{(0-\infty)}$ area under the concentration curve, $C L$ clearance; $V_{\text {ss }}$ steady-state volume of distribution, $L B W$ lean body weight

a The minimum and maximum values are reported if $N \leq 3$; geometric mean and CV\% values are reported if $N>3$, except for $t_{1 / 2}$ for which geometric mean and range are reported

b Ten patients (for $1,800 \mathrm{mg}$ ) and 15 patients (for $420 \mu \mathrm{g} / \mathrm{mL}$ ) were used to calculate the geometric mean for $C_{\max }$

c $C_{\max }$ is reported as received

${ }^{\mathrm{d}}$ Variation in $\mathrm{mg} / \mathrm{kg}$ as the relationship between LBW and $C_{\max }$ is not directly proportional

\section{Overall safety}

The median number of cycles administered was 2 (range 1-18 cycles). The most common grade $3 / 4$ toxicities considered possibly related to study drug were hematologic (thrombocytopenia, anemia, and neutropenia; Table 4). Fifteen patients received one or more transfusions (six red blood cells; seven red blood cells and platelets; one red blood cells, platelets, and plasma; and one plasma).

Of the six patients who died during the study, one patient in cohort 8 died because of complications related to bone marrow suppression (febrile neutropenia and ARDS during cycle 1), and one patient in cohort 11 died because of an outpatient cardiopulmonary arrest after receiving one cycle of study drug (for which drug relatedness could not be ruled out). An additional patient in cohort 11 died in cycle 2 from NSCLC in the setting of post-obstructive pneumonia; the patient had concurrent grade 2 neutropenia that was considered possibly related to study drug. All other patients died because of causes related to their malignancy.

Six patients (cohort 7 or above) were discontinued from the study because of adverse events considered possibly related to study drug and/or complications: coagulopathy
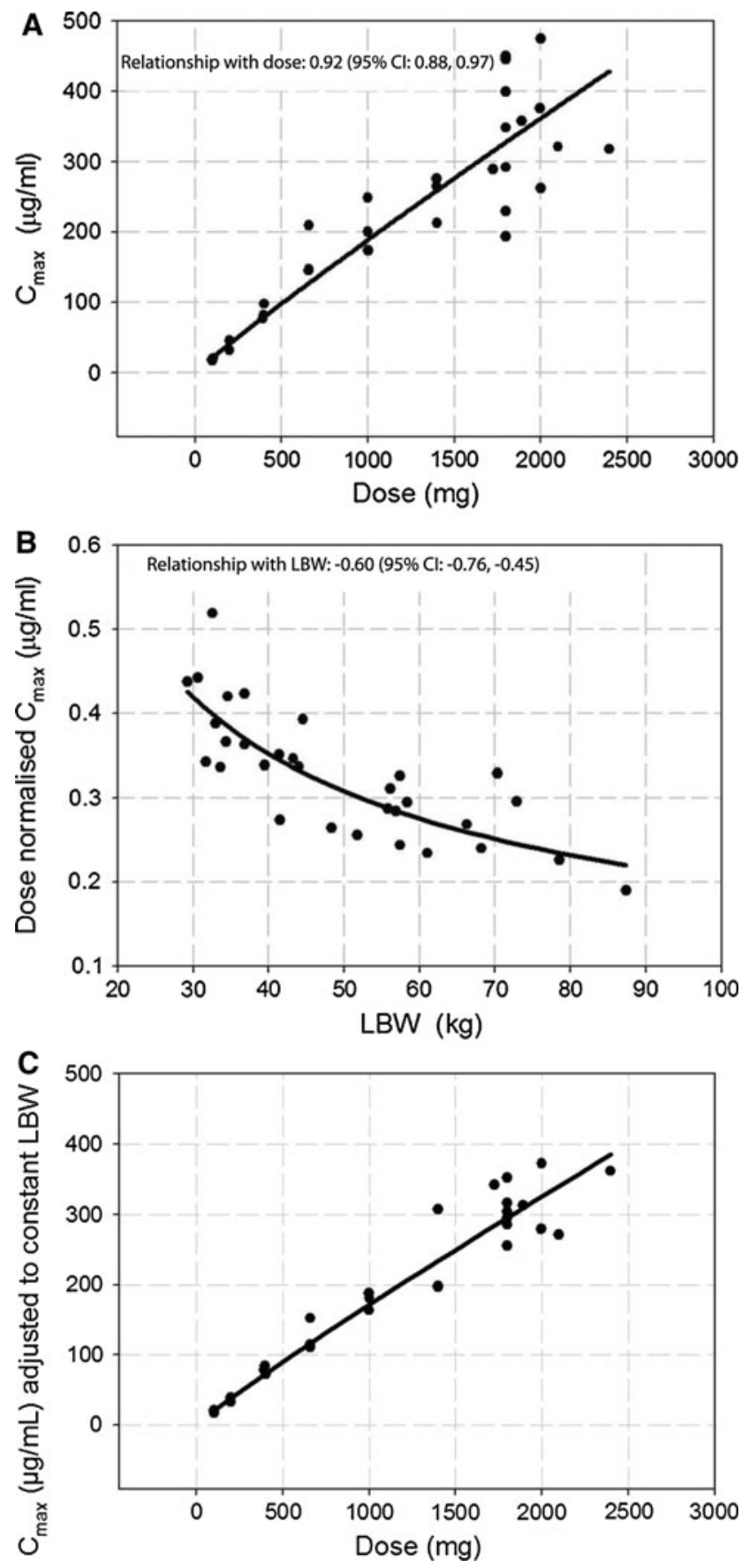

Fig. 1 Results from assessment of dose proportionality showing $C_{\max }$ values increasing less than proportionately with dose (a); the relationship between dose-normalized $C_{\max }$ and LBW, demonstrating an inverse relationship between LBW and $C_{\max }(\mathbf{b})$; and dose proportionality adjusted for LBW, showing reduced interpatient variability of $C_{\max }(\mathbf{c})$. Interpatient $\mathrm{CV} \%$ in $C_{\max }$ after accounting for each covariate (cohorts 1-9): $C_{\max }$ vs. dose: $\mathrm{CV} \%=22 \% ; C_{\max }$ vs. dose + LBW: $\mathrm{CV} \%=13 \%$

with prolonged prothrombin time (patient had been receiving warfarin) associated with rectal bleeding; vaginal bleeding; hypokalemia; thrombocytopenia; febrile neutropenia; and pneumonia and diffuse pulmonary hemorrhage. 
A
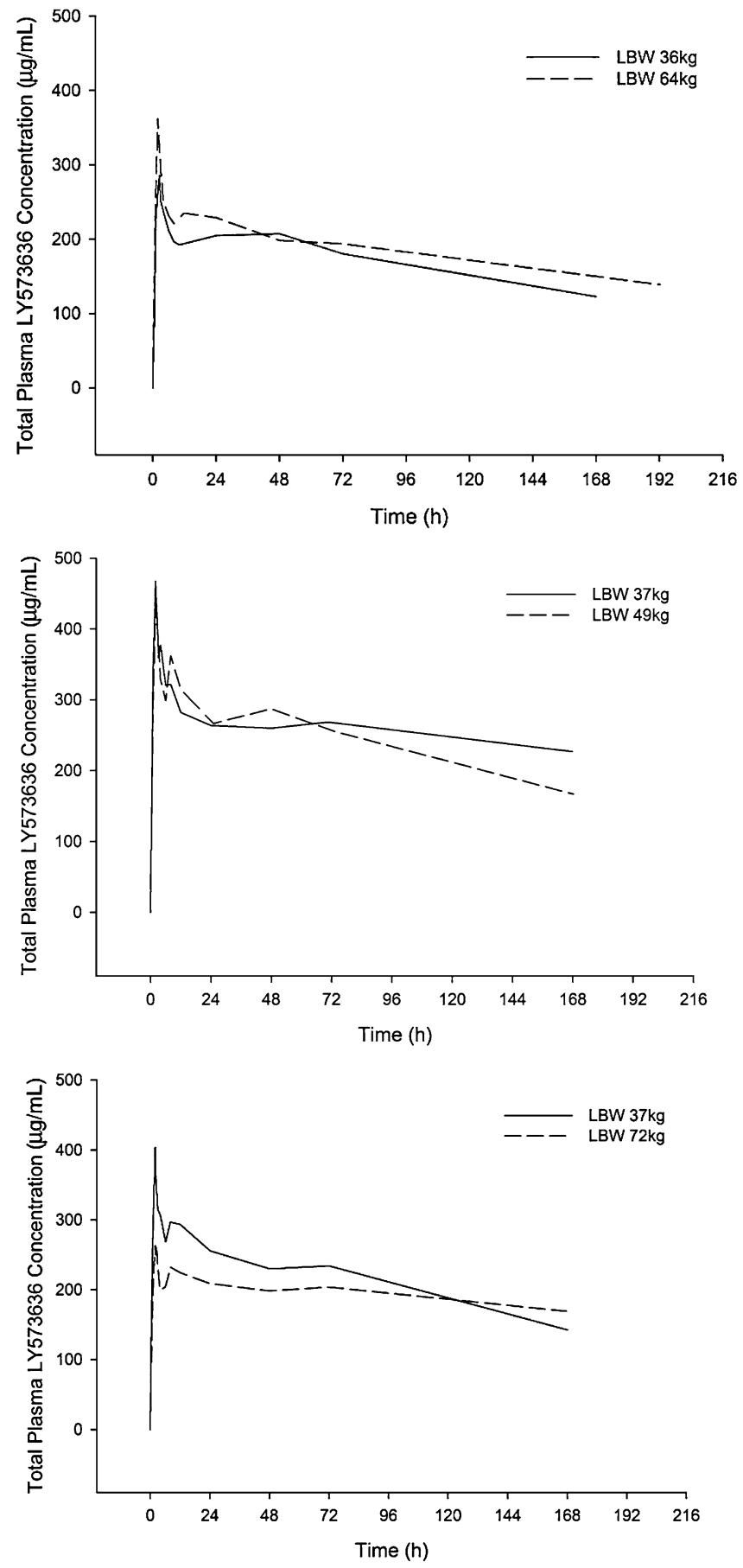

Fig. 2 Total plasma tasisulam (LY573636) concentrations using lean body weight $(L B W)$ and targeted $C_{\max }$ dosing at $420 \mu \mathrm{g} / \mathrm{mL}$ (a) and flat dosing at $1,800 \mathrm{mg}(\mathbf{b})$. The similar differences in LBW indicate that

\section{Efficacy}

Eight patients were discontinued from the study or died before cycle- 2 response assessment because of ARDS and cardiopulmonary arrest (one patient, each); pneumonia and pulmonary hemorrhage (one patient); lost to follow-up (one
B
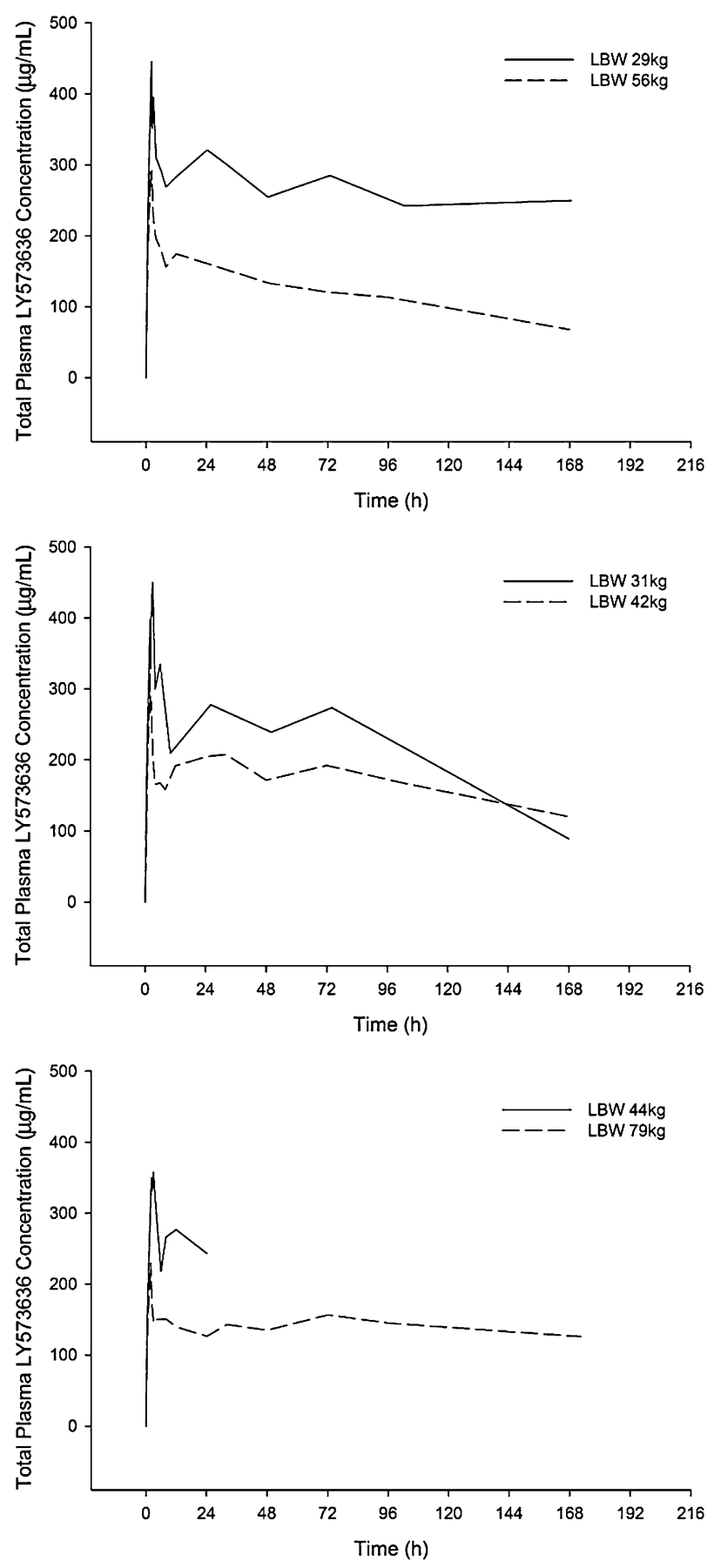

the variability in pharmacokinetic parameters (both AUC and $C_{\max }$ ) was reduced with LBW dosing

patient); withdrawal of consent (one patient); and disease progression (three patients, two of whom were objectively confirmed to have disease progression in follow-up visits). Of the 45 patients assessed for response, there were no objective responses; 25 had progressive disease, 15 had stable disease, and five had an unknown response because of 
Table 4 CTC grade 3 and 4 possibly drug-related toxicities $(N=53)$

\begin{tabular}{lll}
\hline Toxicity & $\begin{array}{l}\text { Grade } 3^{\mathrm{a}} \\
n(\%)\end{array}$ & $\begin{array}{l}\text { Grade } 4 \\
n(\%)\end{array}$ \\
\hline Thrombocytopenia & $7(13.2)$ & $3(5.7)^{\mathrm{b}}$ \\
Anemia & $5(9.4)$ & $1(1.9)$ \\
Neutropenia & $2(3.8)$ & $4(7.5)^{\mathrm{c}}$ \\
Leukopenia & $2(3.8)$ & $1(1.9)$ \\
Hemorrhage/bleeding & $4(7.5)$ & 0 \\
Hypokalemia & $4(7.5)$ & 0 \\
Prothrombin time increased & \\
Diarrhea & $2(3.8)$ & 0 \\
Epistaxis & $2(3.8)$ & 0 \\
Cardiopulmonary arrest & $2(3.8)$ & 0 \\
Adult respiratory distress syndrome & 0 & $1(1.9)$ \\
Febrile neutropenia & 0 & $1(1.9)$ \\
Pneumonia & $1(1.9)$ & $1(1.9)$ \\
\hline
\end{tabular}

CTC Common Toxicity Criteria (Version 2.0, 1998)

a Additional grade 3 toxicities observed in one patient include lymphopenia, alkaline phosphatase, alanine transferase, asparate transferase, abdominal pain/cramping, infection, nausea, vomiting, pleural effusion, and stomatitis/pharyngitis

${ }^{b}$ Platelet recovery times were available for two patients with platelet count nadirs $<15 \times 10^{3} / \mu \mathrm{L}$, and were approximately 11 days for each

c Absolute neutrophil count (ANC) recovery times were available for two patients with ANC nadirs of approximately $0.2 \times 10^{3} / \mu \mathrm{L}$, and were 12 and 14 days

d The prolonged prothrombin time of one patient was associated with grade 3 rectal bleeding/hematochizia and that of the other was a DLT without significant bleeding; both patients were co-administered warfarin

incomplete evaluation. However, five patients who received extensive prior therapy (median 6, range 5-12 prior cancer treatments) for NSCLC (two patients), ovarian cancer (two patients), or thymoma (one patient) received at least 10 cycles of tasisulam (minimum of 7 months) before disease progression.

\section{Discussion}

This is a first-in-human, phase I, dose-escalation study of tasisulam, a novel anticancer agent with a potentially unique dual-faceted mechanism of action (apoptosis via intrinsic mitochondrial death pathway and anti-angiogenic activity). The recommended phase II dosing regimen for tasisulam, administered as an intravenous 2-h infusion on day 1 of 21-day cycles, was identified as a targeted $C_{\max }$ of $420 \mu \mathrm{g} / \mathrm{mL}$ (based on LBW). The most common toxicities considered possibly related to study drug were those associated with bone marrow suppression, specifically, thrombocytopenia and neutropenia. There was no grade $3 / 4$ renal toxicity or neuropathy. Grade 3 nausea, vomiting, and stomatitis were observed in one patient each.
Extensive pharmacokinetic analysis of the flat doses (100-2,400 mg) administered to the first 34 patients revealed wide variability in tasisulam plasma concentrations $\left(C_{\max }\right)$. Preclinical animal studies suggested a correlation between plasma $C_{\max }$ and toxicity, which provided the rationale for identifying covariates that affected $C_{\max }$ in humans. LBW was identified in this study as an important covariate that significantly reduced interpatient variability in $C_{\max }$. These findings led to a novel dosing strategy using LBW to achieve specific $C_{\max }$ targets. Despite the novelty of this dosing schema, dose calculation proved straightforward in this clinical study. Investigators entered patient height, weight, sex, and cycle number into a CD-ROMbased dosing calculator to determine the appropriate patient dose.

Laboratory studies performed on human plasma and serum confirmed that approximately $99.7 \%$ of tasisulam is bound to albumin and may be saturable across the presumed therapeutic dose range. Tasisulam showed bi-exponential disposition with a median terminal elimination half-life of approximately 11 days. Therefore, given the extensive albumin binding of tasisulam and its long terminal half-life, a loading dose in cycle 1 followed by a lower chronic dose in subsequent cycles was implemented to decrease the risk of a progressive rise in drug concentrations in later cycles, which could increase the risk of saturating albumin binding.

Although response was not a primary study objective, 15 of the 45 patients (33\%) assessed for response at the end of cycle 2 ( $28 \%$ of all patients dosed) had stable disease as the best response despite having been heavily pretreated for various refractory solid tumors. In addition, five patients received at least 10 cycles of tasisulam before disease progression. The results of this trial and the findings from another phase I trial evaluating tasisulam [12] led to the initiation of phase II studies in relapsed/refractory solid tumors, including NSCLC, platinum-resistant ovarian cancer [13], soft tissue sarcoma, and malignant melanoma [14]. Pharmacokinetic and safety data from these phase II studies will provide additional opportunities to identify other potentially important laboratory or pharmacokinetic factors that could optimize dosing. In addition, preclinical and clinical investigations continue in an effort to provide additional insights on the mechanism of action of tasisulam.

In summary, analysis of pharmacokinetic and safety in this phase I study allowed for the development of a novel dosing algorithm for tasisulam, a first-in-class anticancer compound with a unique mechanism of action. Implementation of a LBW-based, targeted $C_{\max }$ dosing regimen and a loading/chronic dosing paradigm enabled the determination of the phase II recommended dose for tasisulam, despite pharmacological challenges posed by a high degree of high-affinity albumin binding and a long terminal half-life. 
Although this study was not designed to assess efficacy, stable disease was observed in a reasonable proportion of extensively pretreated patients with solid tumors, suggesting the possibility of antineoplastic activity for tasisulam; testing of this hypothesis is being pursued in currently ongoing phase II clinical trials.

Acknowledgments The authors respectfully thank Noelle Gasco and Kay Chow of Eli Lilly and Company for editing and providing pharmacokinetic data support of this manuscript, respectively, and Michelle R. Burton, R.N., of H. Lee Moffitt Cancer Center, for her work as the study coordinator. Eli Lilly and Company provided the funding for this study.

Open Access This article is distributed under the terms of the Creative Commons Attribution Noncommercial License which permits any noncommercial use, distribution, and reproduction in any medium, provided the original author(s) and source are credited.

\section{References}

1. Corbett TH, Valeriote FA, Polin L et al (1992) Discovery of solid tumor active agents using a soft-agar-colony-formation disk-diffusion-assay. In: Valeriote FA, Corbett TH, Baker LH (eds) Cytotoxic anticancer drugs: models and concepts for drug discovery and development. Kluwer, Norwell, pp 35-87

2. Paull KD, Shoemaker RH, Hodes L, Monks A, Scudiero DA, Rubinstein L, Plowman J, Boyd MR (1989) Display and analysis of patterns of differential activity of drugs against human tumor cell lines: development of mean graph and COMPARE algorithm. J Natl Cancer Inst 81:1088-1092

3. Haritunians T, Gueller S, O' Kelly J, Ilaria R Jr, Koeffler HP (2008) Novel acyl sulfonamide LY573636-sodium: effect on hematopoietic malignant cells. Oncol Rep 20:1237-1242

4. Ratain MJ, Mick R, Schilsky RL, Siegler M (1993) Statistical and ethical issues in the design and conduct of phase I and II clinical trials of new anticancer agents. J Natl Cancer Inst 85:1637-1643
5. Therasse P, Arbuck SG, Eisenhauer EA, Wanders J, Kaplan RS, Rubinstein L, Verweij J, Van Glabbeke M, van Oosterom AT, Christian MC, Gwyther SG (2000) New guidelines to evaluate the response to treatment in solid tumors, European Organization for Research and Treatment of Cancer, National Cancer Institute of the United States, National Cancer Institute of Canada. J Natl Cancer Inst 92:205-216

6. Smith BP, Vandenhende FR, DeSante KA, Farid NA, Welch PA, Callaghan JT, Forgue ST (2000) Confidence interval criteria for assessment of dose proportionality. Pharm Res 17:1278-1283

7. Akaike H (1974) A new look at the statistical model identification. IEEE Trans Auton Control 19:716-723

8. Rowland M, Tozer TN (1995) Clinical pharmacokinetics: concepts and applications, 3rd edn. Lippincott Williams \& Wilkins, Philadelphia, p 149

9. Sterling K (1951) The turnover rate of serum albumin in man as measured by I131-tagged albumin. J Clin Invest 30:1228-1237

10. Janmahasatian S, Duffull SB, Ash S, Ward LC, Byrne NM, Green B (2005) Quantification of lean bodyweight. Clin Pharmacokinet 44:1051-1065

11. Green B, Duffull SB (2004) What is the best size descriptor to use for pharmacokinetic studies in the obese? Br J Clin Pharmacol 58:119-133

12. Slapak CA, LoRusso PM, Mendelson D, Sykes AK, de Alwis DP, Wagner MM, Ilaria RL, Gordon MS (2007) Phase 1 study of LY573636-sodium, an acylsulfonamide anti-cancer compound with a novel mechanism of action, administered as 24-hour continuous infusion in patients with advanced solid tumors. J Clin Oncol 25:107s (suppl 18s; abstr 2542)

13. Gordon M, McMeekin S, Temkin S, Tew W, Yapp S, Scambia G, Streltsova O, Kaiser C, Ilaria R, Look K (2009) A phase II, singlearm study of tasisulam sodium (LY573636 sodium) as 2nd-4th line therapy for platinum-resistant ovarian cancer. AACR-NCIEORTC international conference: molecular targets and cancer therapeutics. Mol Cancer Ther 8(12 suppl):B197

14. Kirkwood JM, Gonzalez R, Reintgen DS, Clingan PR, McWilliams RR, Ilaria RL, de Alwis DP, Zimmermann A, Brown MP, Millward M (2010) A phase II study of tasisulam sodium (LY573636) as second-line treatment for patients with unresectable or metastatic melanoma. J Clin Oncol 28:7s (suppl; abstr 8541) 